\title{
SYNTHESIS AND STABILITY TEST OF RESVERATROL-CONJUGATED GOLD NANOPARTICLE WITH POLYVINYL ALCOHOL STABILIZATION
}

\author{
SUTRIYO SUTRIYO*, RADITYA ISWANDANA, MARINA DWI HAFSHARI
}

Laboratory of Pharmaceutics and Pharmaceutical Technology, Faculty of Pharmacy, Universitas Indonesia, Depok 16424, Indonesia. Email: sutriyo@farmasi.ui.ac.id

Received: 02 October 2019, Revised and Accepted: 24 December 2019

\section{ABSTRACT}

Objective: Gold nanoparticles (AuNPs) have been developed as a promising effective site-specific drug to increase drug efficacy and reduce potential side effects. However, AuNPs are unstable because they easily aggregate. This study aims to produce stable resveratrol (RSV)-conjugated AuNPs using polyvinyl alcohol (PVA).

Methods: AuNPs were synthesized using the Turkevich method, which involves the reduction of chloroauric acid with sodium citrate as a reductor. AuNPs were then modified with PVA as a stabilizing agent and conjugated with RSV as a drug model in the carrier system. The formed conjugates were characterized using ultraviolet-visible spectrophotometry, Fourier transform infrared spectroscopy, particle size analysis, and high-performance liquid chromatography. Furthermore, stability tests were performed in various media (2\% bovine serum albumin [BSA], 1\% cysteine, phosphatebuffered saline [PBS] pH 4, PBS pH 7.4, and 0.9\% NaCl) for 28 days.

Results: RSV-AuNPs-PVA had a particle size of $78.75 \mathrm{~nm}$, with polydispersity index (PDI) of 0.356 , zeta potential of $-36.1 \mathrm{mV}$, and highest entrapment efficiency of 78.1\% \pm 0.7 . RSV-AuNPs without PVA stabilization had a particle size of $51.97 \mathrm{~nm}$, with PDI of 0.694 and zeta potential of $-24.6 \mathrm{mV}$. The results of the stability tests demonstrated that RSV-AuNPs-PVA was stable in 2\% BSA, PBS pH 7.4, PBS pH 4, and NaCl $0.9 \%$ and were unstable in $1 \%$ cysteine. RSV-AuNPs without PVA were stable in 2\% BSA and PBS pH 7.4 and unstable in $1 \%$ cysteine, PBS pH 4, and $0.9 \% \mathrm{NaCl}$.

Conclusion: PVA can improve the physical stability of RSV-AuNPs conjugates.

Keywords: Conjugate, Gold nanoparticles, Polyvinyl alcohol, Resveratrol, Stability.

(c) 2020 The Authors. Published by Innovare Academic Sciences Pvt Ltd. This is an open access article under the CC BY license (http://creativecommons. org/licenses/by/4. 0/) DOI: http://dx.doi.org/10.22159/ijap.2020.v12s1.FF049

\section{INTRODUCTION}

Nanomaterial preparations using different noble metals are used in a wide variety of biomedical applications due to their unique physical and chemical properties; for example, as drug carriers for targeted delivery in cancer treatment, for molecular imaging, gene therapy, and in other applications, such as biosensors [1]. Nanoparticles have several advantages, such as improvement in the intracellular infiltration, circulation time of the drug, and enhanced hydrophobic solubility [2]. Nanoparticles are usually $<100 \mathrm{~nm}$ in size. They consist of different biodegradable materials such as natural or synthetic polymers, lipids, or metals [3]. Gold nanoparticles (AuNPs) have a great potential to be used in effective targeted drug delivery because they are easily synthesized, biocompatible, nontoxic, and, due to their negative charge, can be easily functionalized by all kinds of biomolecules [4]. In general, AuNPs are synthesized by the chemical reduction of chloroauric acid $\left(\mathrm{HAuCl}_{4}\right)$ using reducing agents, such as sodium citrate and sodium borohydride [5]. AuNPs have sizes ranging from 1 to $100 \mathrm{~nm}$ [6]. Although gold appears yellow, at the nanoscale level, it can appear red, purple, or blue, based on the size of the AuNP [7].

AuNPs are generally unstable due to their high surface energy and can often aggregate due to the high ionic strength of many biological fluids [8]. Therefore, a suitable stabilizer should be added to prevent aggregation. Polyvinyl alcohol (PVA) acts as a stabilizer to prevent aggregation by decreasing the surface energy of AuNPs and minimizing Van der Waals attraction [9]. PVA has remained a promising choice for AuNP stabilization due to its biocompatibility, water solubility, and hydroxyl fill nature [10].

Resveratrol (RSV) (3,5,4'-trihydroxystilbene) is a polyphenol compound found in different medicinal plants. RSV has antioxidant, anti-inflammatory, and anti-senescence properties, and potential as an anticancer drug. It inhibits cell growth, tumor proliferation, invasion, metastasis, and angiogenesis by downregulating many molecular signaling targets [11]. RSV-AuNP can inhibit the signaling of molecules involved in breast cancer invasions, such as MMP-9, COX-2, NF- $\mathrm{KB}$, and AP-1 [12]. The inhibitory effect is stronger than that of a single RSV molecule at the same concentration. Thus, RSV has been potentially developed as a drug model with anticancer activity.

In this research, the aim was to produce a PVA-stabilized AuNP as a targeted drug delivery system with RSV as a drug model. The formed RSV-AuNPs-PVA was evaluated by physical stability tests and characterized by ultraviolet (UV)-visible spectrophotometry, Fourier transform infrared (FTIR) spectroscopy, particle size analysis, and highperformance liquid chromatography (HPLC).

\section{MATERIALS AND METHODS}

\section{Materials}

RSV, chloroauric acid, PVA (Mowiol ${ }^{\circledR} 8-88$, Mw 67,000), phosphatebuffered saline (PBS), cysteine, and bovine serum albumin (BSA) were purchased from Sigma-Aldrich (St. Louis, MO, USA). Sodium chloride, methanol, ethanol, acetonitrile, and sodium citrate were purchased from Merck (Germany). Distilled water and aqua bidest were also used.

\section{Synthesis of RSV-AuNPs-PVA and RSV-AuNPs}

The aqueous solution of $\mathrm{HAuCl}_{4} \cdot 3 \mathrm{H}_{2} \mathrm{O}(1 \mathrm{mM}) 25.0 \mathrm{~mL}$ was heated to approximately $95^{\circ} \mathrm{C}$ with stirring at $350 \mathrm{rpm}$. A volume of $0.846 \mathrm{ml}$ of $130 \mathrm{mM}$ aqueous sodium citrate solution was rapidly added to the solution with stirring. The color of the solution changed instantly from pale yellow to colorless, then turned to dark blue and reddish-purple 
after a few minutes. Stirring and heating were maintained for $15 \mathrm{~min}$ after the addition of sodium citrate. The heat was then removed, and the solution was stirred on the addition of $0.833 \mathrm{ml}$ of $3 \% \mathrm{w} / \mathrm{v}$ PVA to the reddish-purple solution. The solution was stirred at $350 \mathrm{rpm}$ and cooled at room temperature. Finally, $5.7 \mathrm{ml}$ aqueous RSV solution (10 ppm) was added drop-by-drop to the solution and continuously stirred at $150 \mathrm{rpm}$ for $2 \mathrm{~h}$ at room temperature to obtain PVA-stabilized, resveratrol-conjugated gold nanoparticles (RSV-AuNPs-PVA). The RSV-AuNPs solution was produced by the same method, but without the addition of PVA

\section{Characterization}

Physical appearance of RSV-AuNPs and RSV-AuNPS-PVA

The physical appearance of RSV-AuNPs and RSV-AuNPs-PVA was analyzed by physical comparison to observe the differences in color and the presence/absence of precipitates at the bottom of the vial.

Measurement of absorbance and wavelength under UV-visible spectrophotometry

The absorbance spectrum of RSV-AuNPs and RSV-AuNPs-PVA with comparison 1:10 in aquadest was recorded using a UV-visible spectrophotometer UV-1800 (Shimadzu, Japan) in the range of 400$800 \mathrm{~nm}$.

\section{Infrared spectrum analysis using FTIR spectroscopy}

The FTIR spectra of standard RSV and standard PVA were determined, and the dried samples were ground homogeneously with potassium bromide. The infrared spectrum analysis was recorded on an FTIR8400 S (Shimadzu, Japan) in a wavenumber region of $400-4000 \mathrm{~cm}^{-1}$.

Particle size distribution, polydispersity index (PDI), and zeta potential The particle size distribution, PDI, and zeta potential of the RSVAuNPs and RSV-AuNPs-PVA were determined by dynamic light scattering using a Malvern Zetasizer (Nano ZS, Malvern Instruments, Worcestershire, UK).

\section{RSV entrapment efficiency}

A standard RSV solution of 1000 ppm was prepared in methanol, then diluted to $1,5,10,15,20$, and $25 \mathrm{ppm}$ to produce a calibration curve. Approximately $2 \mathrm{ml}$ of RSVAuNPsPVA was centrifuged at 12,000 rpm at $27^{\circ} \mathrm{C}$ for $60 \mathrm{~min}$. The supernatant was then filtered through a using a filter with a pore size of $0.22 \mu \mathrm{m}$ and analyzed indirectly using HPLC. The amount of RSV trapped in the RSV-AuNPs-PVA was determined by subtracting the quantity in the supernatant from the total quantity used during the preparation. These analyses were performed in triplicate. The entrapment efficiency of RSV was calculated using the equation:

$$
\mathrm{EE}(\%)=\frac{\text { TotalRSV }- \text { FreeRSV }}{\text { TotalRSV }} \times 100 \%
$$

Chromatographic conditions [10]

Mobile phase $=$ aqua pro injection: acetonitrile (3:1), then glacial acetic acid added until $\mathrm{pH} 3$

Flow rate $=1.5 \mathrm{ml} / \mathrm{min}$

Injection volume $=20 \mu \mathrm{l}$

Detector $=$ UV-visible at $306 \mathrm{~nm}$

Column $=\mathrm{C} 18$.

\section{In vitro stability tests in various media}

Typically, $2 \mathrm{ml}$ of sample was redispersed to $1.0 \mathrm{ml}$ of each of the following media: $2 \%$ BSA, $1 \%$ cysteine, $0.9 \% \mathrm{NaCl}$, PBS pH 4 , and PBS $\mathrm{pH}$ 7.4. The stability test was carried out for $28 \mathrm{~d}$ because the clearance of AuNPs took 28 days. In the $1^{\text {st }}$ week, samples were taken daily and, for the next $3 \mathrm{w}$ weeks, every 3 days. The stability of AuNPs was monitored by recording the absorbance at 400-800 $\mathrm{nm}$ with UV-visible spectrophotometry. The results were generated by observation of the color change and the precipitates formed absorbance change, maximum wavelength shift, and the change in full width at half maximum (FWHM) value.

\section{RESULTS}

Synthesis of AuNP and optimization concentration of PVA as a stabilizing agent

The chemical reduction of $\mathrm{HAuCl}_{4} \cdot 3 \mathrm{H}_{2} \mathrm{O}$ is widely used for the synthesis of AuNPs. Sodium citrate $\left(\mathrm{Na}_{3} \mathrm{C}_{6} \mathrm{H}_{5} \mathrm{O}_{7}\right)$ acts as a reducing agent, where $\mathrm{Au}^{3+}$ is reduced to $\mathrm{Au}^{0}$ to form AuNPs. The formation of AuNPs can be observed by a change in color from pale yellow to reddish-purple. This color change occurs due to surface plasmon resonance, which is a specific property of AuNPs. The spectra exhibit an absorption band at $528.5 \mathrm{~nm}$, which is a typical plasmon band of AuNPs, indicating that AuNPs have formed. The maximum AuNPs absorbance occurred at wavelengths from 520 to $565 \mathrm{~nm}$, which corresponds to green and purple (Fig. 1) [13].

In the preparation of RSV-AuNPs-PVA, first, the optimization of PVA concentration as a stabilizer was determined by varying the concentration of PVA $(1 \%, 3 \%, 5 \%, 6 \%$, and $7 \%)$ and observing the absorbance change over 7 days using a UV-visible spectrophotometer. The obtained AuNPs stabilized by varying concentrations of PVA were then diluted with a ratio of 1:10 using aquadest. The results are shown in Fig. 2.

At a concentration of $1 \%$, PVA showed the lowest absorbance, which continued to decrease until day 7 . The absorbance results showed that the 3\% PVA concentration had the highest absorbance and the most stable UV-visible results over 7 days, while the preparations of AuNPsPVA with higher concentrations of PVA (5\%, 6\%, and 7\%) showed lower absorbance and fluctuating graphs. Therefore, the optimal condition for stabilized AuNPs was determined to be at a PVA concentration of $3 \%$.

\section{Characterization of RSV-AuNPs and RSV-AuNPs-PVA}

Physical appearance

The obtained nanoparticle solution was a reddish-purple color, as shown in Fig. 3. All the AuNPs were prepared as a clear solution, which indicates that the Au was homogenously dispersed [13]. The RSVAuNPs had a dark purple color (Fig. 3a), while the RSV-AuNPs-PVA had a clear reddish-purple color (Fig. 3b). These color differences were found to correlate with the size of the particles formed. The reddishpurple color signifies that the particles have a small particle size, and

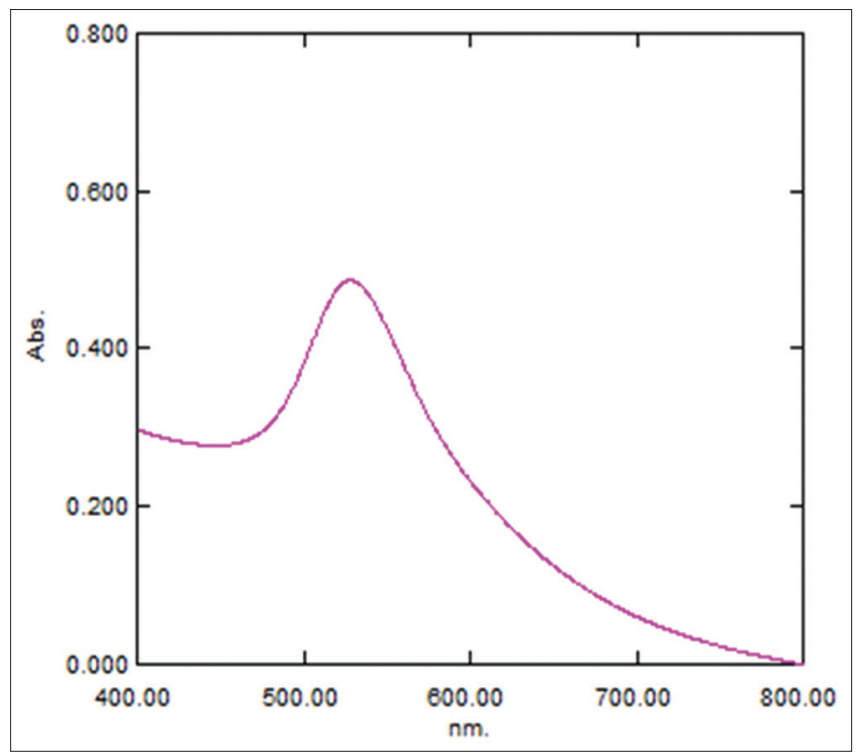

Fig. 1: Ultraviolet-visible absorption spectrum $(528.50 \mathrm{~nm})$ of gold nanoparticle 
the darker purple or blue color signifies a larger particle size [14]. Larger particles form due to the tendency of aggregation. This result showed that the RSV-AuNPs-PVA had a smaller particle size than RSV-AuNPs, indicating that PVA acts as a steric stabilizer by creating a repulsive barrier to prevent aggregation.

Measurement of absorbance and wavelength using UV-visible spectrophotometer

In this study, we observed the absorbance of $\mathrm{HAuCl}_{1} 1 \mathrm{mM}, 10 \mathrm{ppm}$ RSV standard, 3\% PVA solution, RSV-AuNPs, and RSV-AuNPs-PVA. The UVvisible spectra of the $\mathrm{HAuCl} 4$ solutions before and after the reduction of metal ions were measured. In the aqueous solution before reduction, $\mathrm{HAuCl}_{4}$ showed a strong absorption band at $290 \mathrm{~nm}$ due to charge transfer between the metal and chloro ligands [15]. After reduction with sodium citrate, the $290 \mathrm{~nm}$ band of $\mathrm{AuCl}^{4-}$ vanished, indicating that $\mathrm{AuCl}^{4-}$ was completely reduced. Instead, we observed a new absorption band at $529 \mathrm{~nm}$ (RSV-AuNPs-PVA) and $534 \mathrm{~nm}$ (RSV-AuNPs), which could be assigned to a plasmon band of the AuNPs. The PVA solution provided maximum absorption at $279.40 \mathrm{~nm}$ (Fig. 4)

The maximum absorbance of RSV-AuNPs-PVA was 0.374, higher than that of RSV-AuNPs (0.260). The absorbance showed a similar trend with the number of AuNPs formed. The higher absorbance indicated that more AuNPs were formed [16], indicating that PVA can stabilize AuNPs by preventing aggregation.

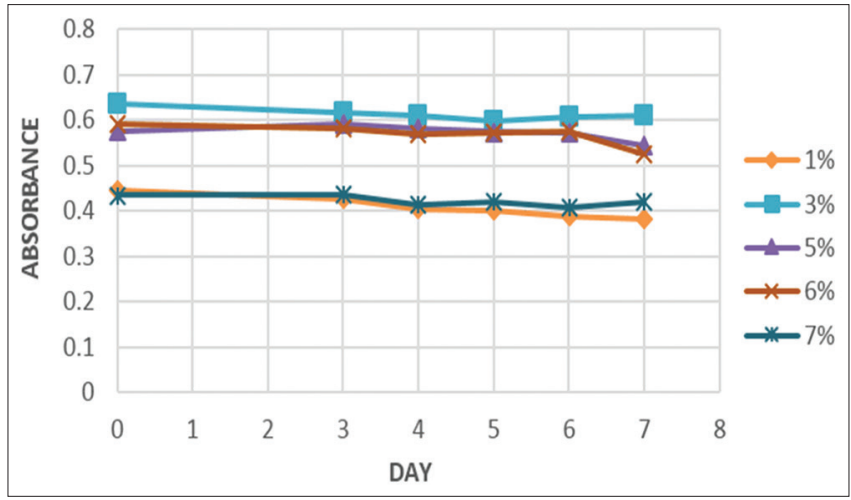

Fig. 2: Optimization concentration of polyvinyl alcohol (PVA) in the preparation of resveratrol-gold nanoparticles-PVA $(400-800 \mathrm{~nm})$

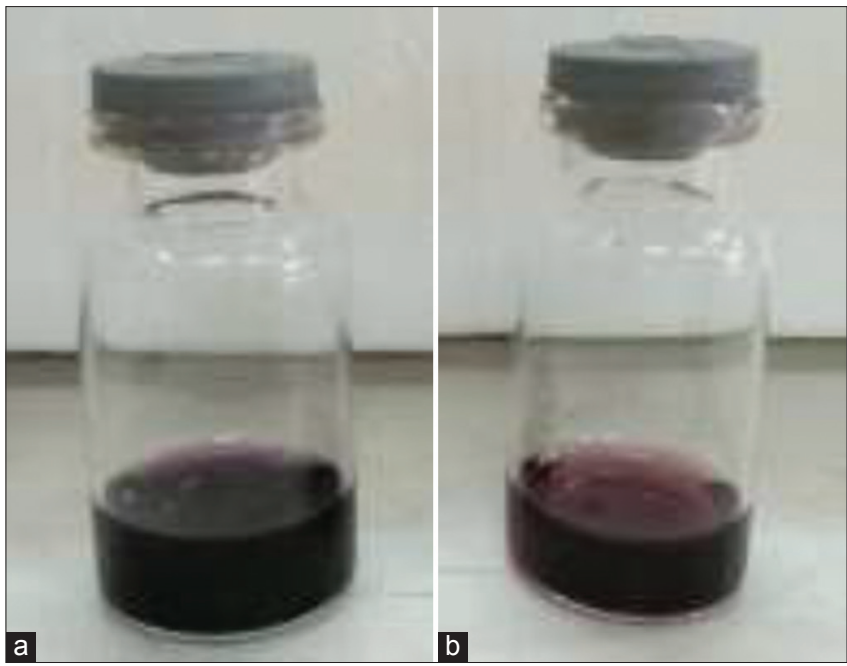

Fig. 3: Physical appearance of (a) resveratrol conjugated gold nanoparticle (RSV-AuNPs), and (b) resveratrol conjugated gold nanoparticle with PVA stabilization (RSV-AuNPs-PVA)
Particle size distribution, PDI, and zeta potential

The results for particle size, PDI, and zeta potential are shown in Table 1. The values suggest that the particle size of both RSV-AuNPs and RSV-AuNPs-PVA was in the nanosize range of 1-100 nm [5]. RSVAuNPs-PVA particles were found to be bigger than RSV-AuNPs particles because dynamic light scattering obtains a hydrodynamic diameter based on the diffusion of the particles. If a gold colloid is coated with a protective stabilizer then the size measured will be a reflection of the core gold and the protective shell; therefore, the particle size is increased.

PDI is a parameter that defines particle size distribution. The PDIs of RSV-AuNPs and RSV-AuNPs-PVA were 0.694 and 0.356, respectively. The closer the PDI value is to zero, the more homogeneous the dispersion of particles in the solution [17]. The PDI reflects the stability of a nanoparticle system, with a higher value indicating increasing particle aggregation and low homogeneity.

The zeta potential of nanoparticles is commonly used to characterize their surface charge properties. A zeta potential between $-30 \mathrm{mV}$ and $30 \mathrm{mV}$ correlates to high stability, as the surface charge prevents aggregation of the particles [18]. The zeta potential of RSV-AuNPs-PVA was $-36.1 \mathrm{mV}$, which is smaller than $-30 \mathrm{mV}$, so the formulation had a good value of zeta potential. The RSV-AuNPs-PVA were found to be more stable than the RSV-AuNPs (zeta potential of $-24.6 \mathrm{mV}$ ), which reached the "threshold of delicate dispersion" category defined by the American Filtration and Separations Society [19].

RSV entrapment efficiency

The RSV entrapment efficiency reflects the amount of RSV entrapped within the AuNPs. The standard calibration curve equation obtained was $y=92620 x-28703$ with $r=0.9998$. The chromatogram showed that the retention times of both standard RSV and RSV in the samples were

Table 1: Physicochemical characterization of gold nanoparticles

\begin{tabular}{llll}
\hline Samples & $\begin{array}{l}\text { Particle size } \\
\text { (nm) }\end{array}$ & $\begin{array}{l}\text { Polydispersity } \\
\text { index }\end{array}$ & $\begin{array}{l}\text { Zeta potential } \\
\text { (mV) }\end{array}$ \\
\hline RSV-AuNPs & 51.97 & 0.694 & -24.6 \\
RSV-AuNPs-PVA & 78.75 & 0.356 & -36.1 \\
\hline
\end{tabular}

RSV: Resveratrol, AuNPs: Gold nanoparticles, PVA: Polyvinyl alcohol

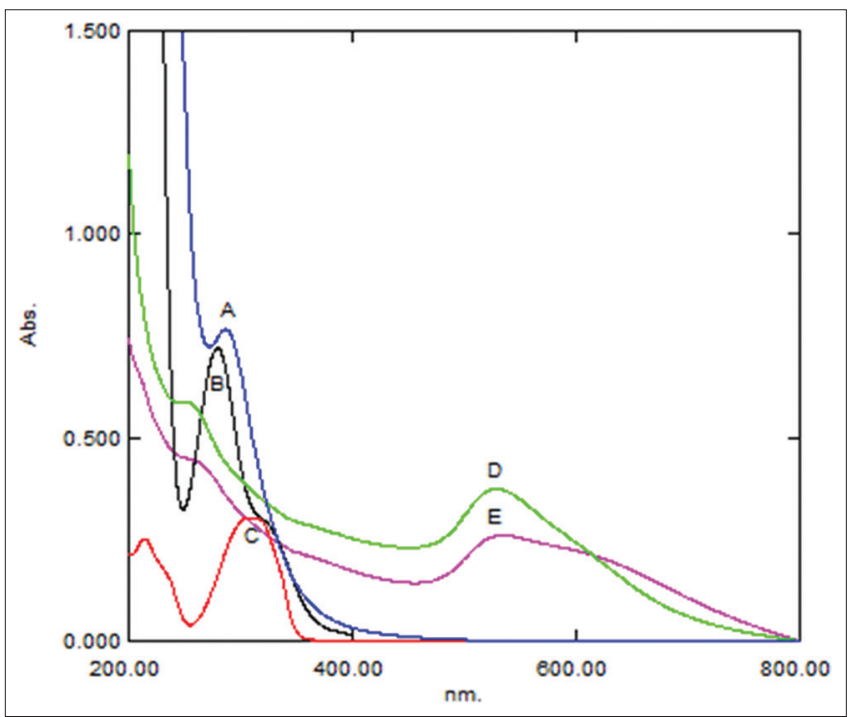

Fig. 4: The spectrum absorbance of $\mathrm{HAuCl} 1 \mathrm{mM}(\mathrm{a}), 3 \%$ polyviny alcohol (PVA) solution (b), 10 ppm resveratrol standard (c), resveratrol-gold nanoparticles (RSV-AuNPs)-PVA (d), RSV-AuNPs (e) 
approximately $14 \mathrm{~min}$. From the results of the calculated data, the mean percentage of RSV entrapment efficiency over three measurements was $78.1 \% \pm 0.7 \%$.

\section{Infrared spectrum analysis}

The FTIR spectra of RSV, PVA, AuNPs, RSV-AuNPs, and RSV-AuNPs-PVA are shown in Fig. 5. RSV exhibited prominent bands at wavelengths of $3380-2925 \mathrm{~cm}^{-1}(\mathrm{O}-\mathrm{H}) ; 1613 \mathrm{~cm}^{-1}$.

( $\mathrm{C}=\mathrm{C}$ Alkene); $1583 \mathrm{~cm}^{-1}$ and $1475 \mathrm{~cm}^{-1}$ ( $\mathrm{C}=\mathrm{C}$ Aromatic); and $833 \mathrm{~cm}^{-1}$ (meta-para substitution). Bands of RSV-AuNP and RSV-AuNPs-PVA were observed at $833 \mathrm{~cm}^{-1}$ (meta-para substitution) and $1583 \mathrm{~cm}^{-1}$ and $1475 \mathrm{~cm}^{-1}$ ( $\mathrm{C}=\mathrm{C}$ Aromatic), suggesting that RSV was present in the samples. The PVA exhibited hydroxyl group $(\mathrm{O}-\mathrm{H})$ prominent bands at a wavelength of $2875.96 \mathrm{~cm}^{-1}(\mathrm{C}-\mathrm{H})$ and strong intensity at a wavelength of $2357.09 \mathrm{~cm}^{-1}$ (vibration of the $\mathrm{O}-\mathrm{H}$ group). The FTIR spectra of RSV-AuNPs-PVA also showed prominent bands at a wavelength of $2875.96 \mathrm{~cm}^{-1}(\mathrm{C}-\mathrm{H})$, while these were absent in the spectra of AuNP and RSV-AuNP. These results suggest that PVA was present in the samples and that interaction took place between AuNP and the PVA at the $\mathrm{O}-\mathrm{H}$ groups.

\section{In vitro stability studies of AuNPs}

In vitro stability analysis of RSV-AuNPs and RSV-AuNPs-PVA without medium (control)

The UV-visible absorbance of RSV-AuNPs-PVA was decreased but not significantly compared to the initial absorbance, while RSV-AuNPs showed fluctuating change (Fig. 6). The change in wavelength over 28 days of the RSV-AuNPs ( $>10 \mathrm{~nm}$ ) indicated the change in particle size in the AuNPs system due to aggregation of the colloid [19]. Furthermore, the absorbance spectrum of the RSV-AuNPs-PVA was narrower than that of the RSV-AuNPs. This indicates that the FWHM value of the RSV-AuNPs-PVA was lower than that of the RSV-AuNPs. The instability of RSV-AuNPs was also supported by their change in color from reddish-purple to clear when precipitates formed on day 16. On day 19, FWHM and wavelength shift could no longer be calculated because the peak line reached point zero. Thus, RSV-AuNPs-PVA were more stable due to the addition of PVA, which prevents aggregation.

In vitro stability analysis of RSV-AuNPS and RSV-AuNPS-PVA in $2 \%$ BSA Both RSV-AuNPs and RSV-AuNPs-PVA showed stable absorbance results (Fig. 7 ) in $2 \%$ BSA. The maximum wavelength shift ( $\lambda$ max) of RSV-AuNPs and RSV-AuNPs-PVA did not exceed $10 \mathrm{~nm}$ after 28 days, indicating the physical stability of the AuNP system. Their stability is also supported by their FWHM values, which did not change significantly. It can be concluded that both RSV-AuNPs and RSV-AuNPs-PVA are stable in $2 \%$ BSA medium.

In vitro stability analysis of RSV-AuNPs and RSV-AuNPs-PVA in $1 \%$ cysteine

RSV-AuNPs were found to unstable in 1\% cysteine medium (Fig. 8). This instability was demonstrated by the change in the color of the RSV-AuNPs solution from reddish-purple to blue; then, $1 \mathrm{~h}$ later, to clear with precipitates at the bottom of the vial. The RSV-AuNPs absorption spectrum was flat from day 0 , and the absorbance was low $(0.028)$. The FWHM value of RSV-AuNPs was high due to its widened spectrum.

RSV-AuNPs-PVA also showed instability in $1 \%$ cysteine medium, demonstrated by its decreased absorbance day-by-day. Moreover, the maximum wavelength shifts were $>10 \mathrm{~nm}$ during the observation period, and higher FWHM values were observed until day 7. Although PVA did not produce great stability in this medium, the results were much better than for RSV-AuNPs. Stabilization of AuNPs with PVA can prevent aggregation in 1\% cysteine medium until day 7 (Fig. 8).

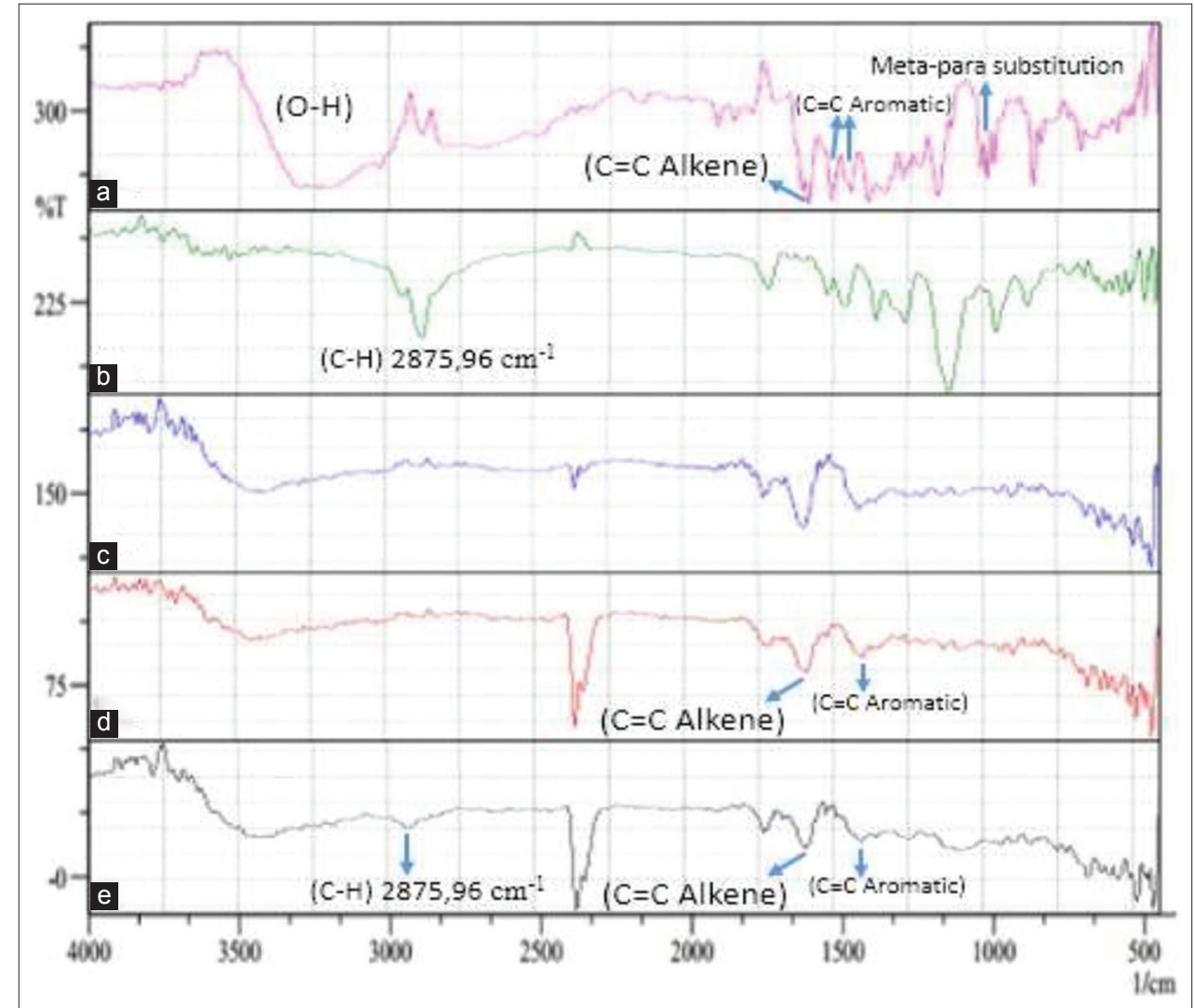

Fig. 5: Fourier transform infrared spectra from top to bottom: resveratrol standard (a), 3\% polyvinyl alcohol (PVA) standard (b), gold nanoparticles (AuNPs) (c), resveratrol (RSV)-AuNPs (d), RSV-AuNPs-PVA (e) 


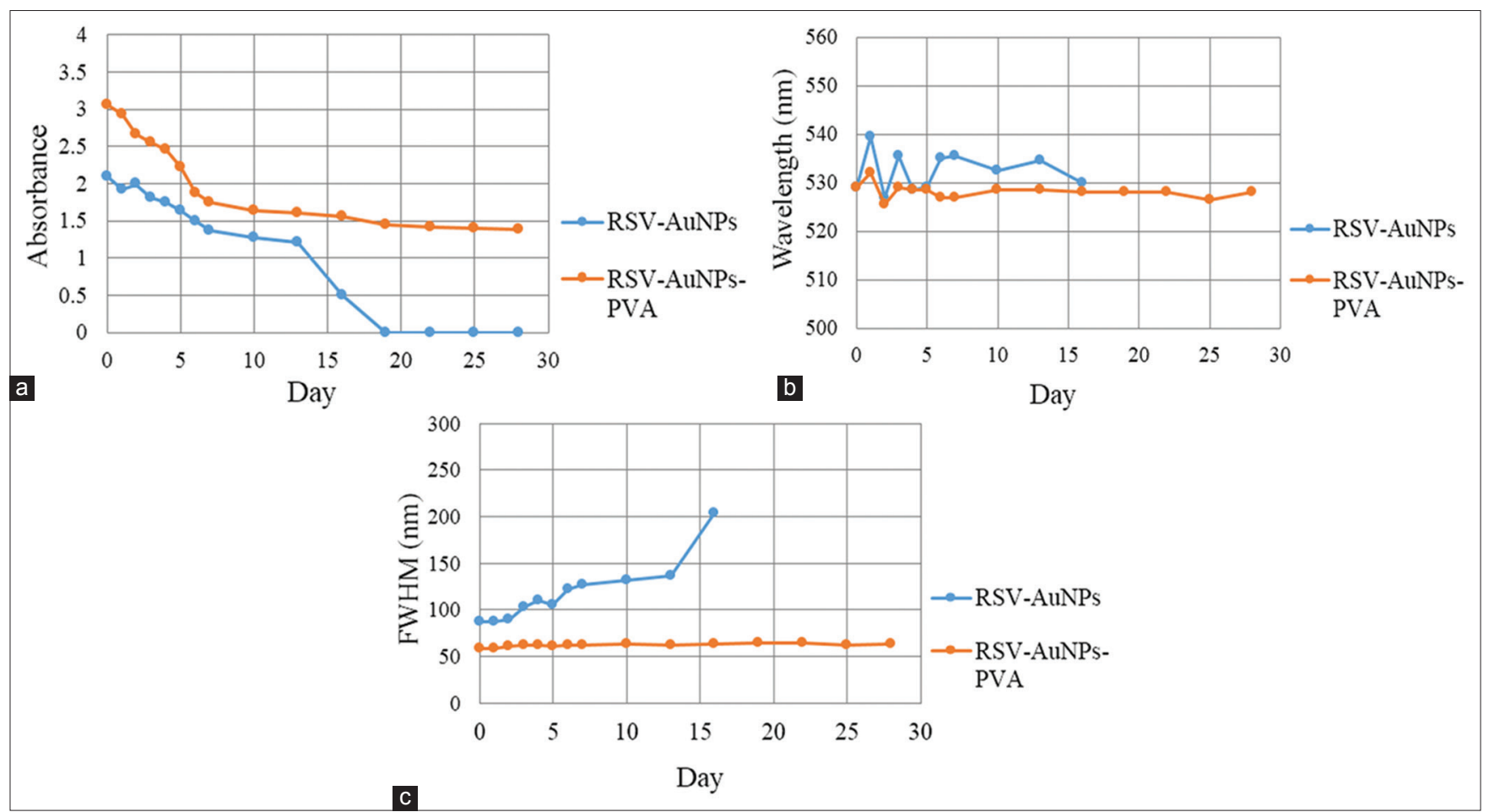

Fig. 6: (a-c) Absorbance comparison graph, maximum wavelength ( $\lambda$ max), and full width at half maximum value between resveratrol-gold nanoparticles (RSV-AuNPs) with RSV-AuNPs-polyvinyl alcohol without medium
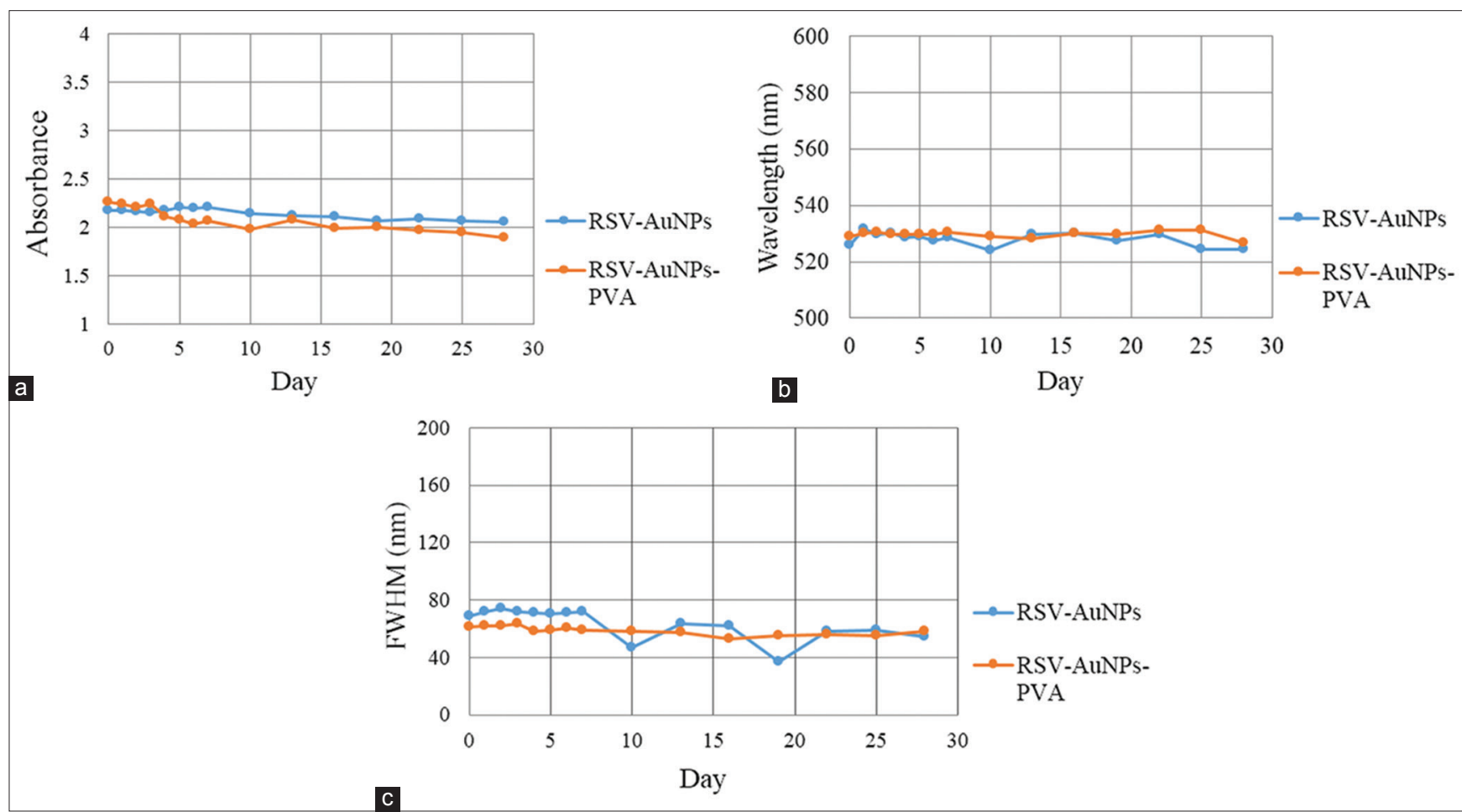

Fig. 7: (a-c) Absorbance comparison graph, maximum wavelength ( $\lambda$ max), and full width at half maximum value between resveratrol-gold nanoparticles (RSV-AuNPs) with RSV-AuNPs-polyvinyl alcohol in $2 \%$ bovine serum albumin

In vitro stability analysis of RSV-AuNPS and RSV-AuNPS-PVA in PBS at various $\mathrm{pH}$ conditions

In PBS pH 7.4 medium, RSV-AuNPs and RSV-AuNPs-PVA tended to be stable (Fig. 9). Based on the UV-visible spectrum, the absorbance values of both RSV-AuNPs and RSV-AuNPs-PVA were relatively stable during the observation period. The changes in the $\lambda$ max of RSV-AuNPs and
RSV-AuNPs-PVA were $<10 \mathrm{~nm}$, and the FWHM values were relatively stable.

In PBS pH four medium, RSV-AuNPs clearly showed instability. This was demonstrated by the color change from reddish-purple to clear with precipitates. From day 0 , the absorption spectrum of RSV-AuNPs was flat in the region of 500-550 nm with a low absorbance value (0.014). 


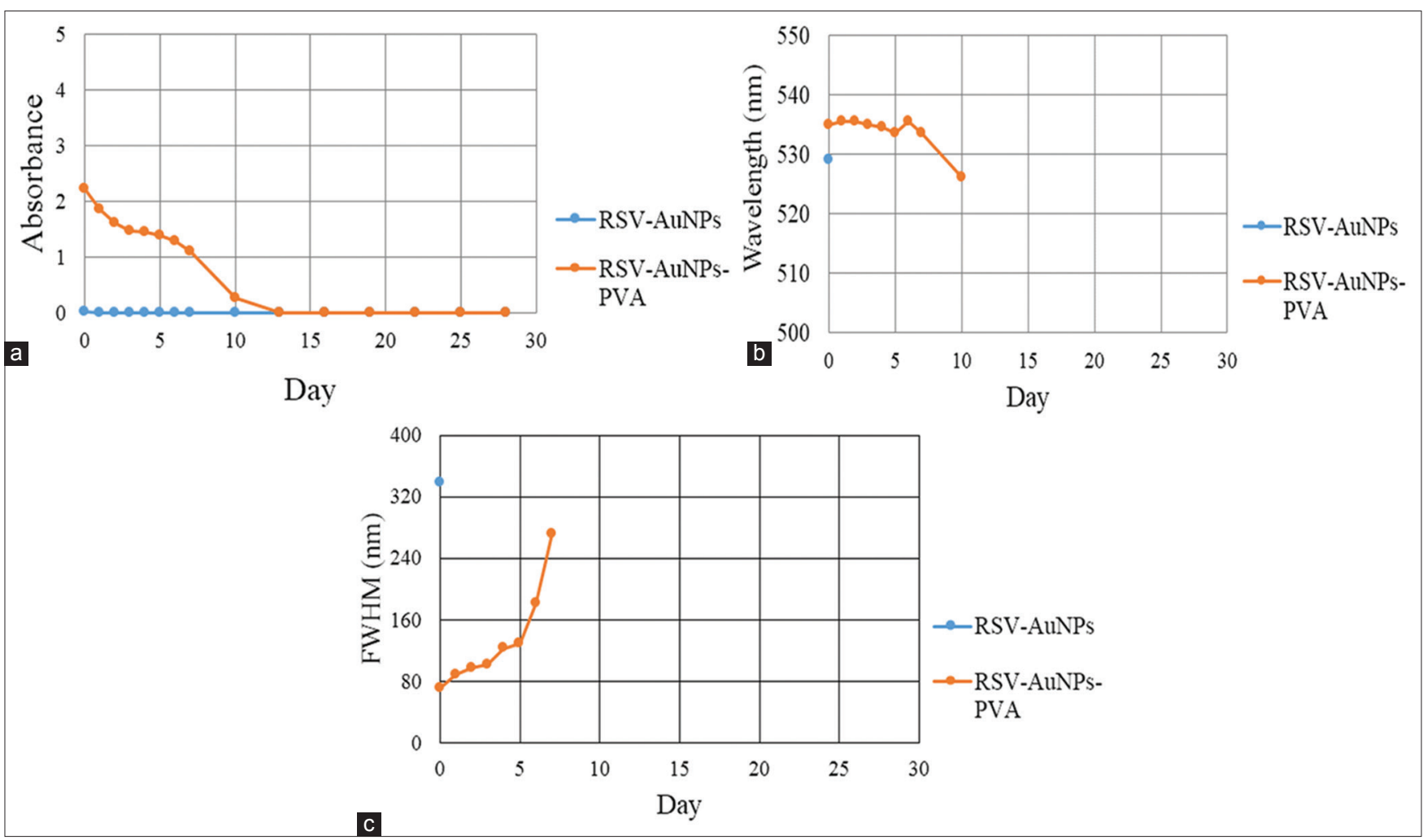

Fig. 8: (a-c) Absorbance comparison graph, maximum wavelength ( $\lambda$ max), and full width at half maximum value between resveratrol-gold nanoparticles (RSV-AuNPs) with RSV-AuNPs-polyvinyl alcohol in 1\% cysteine
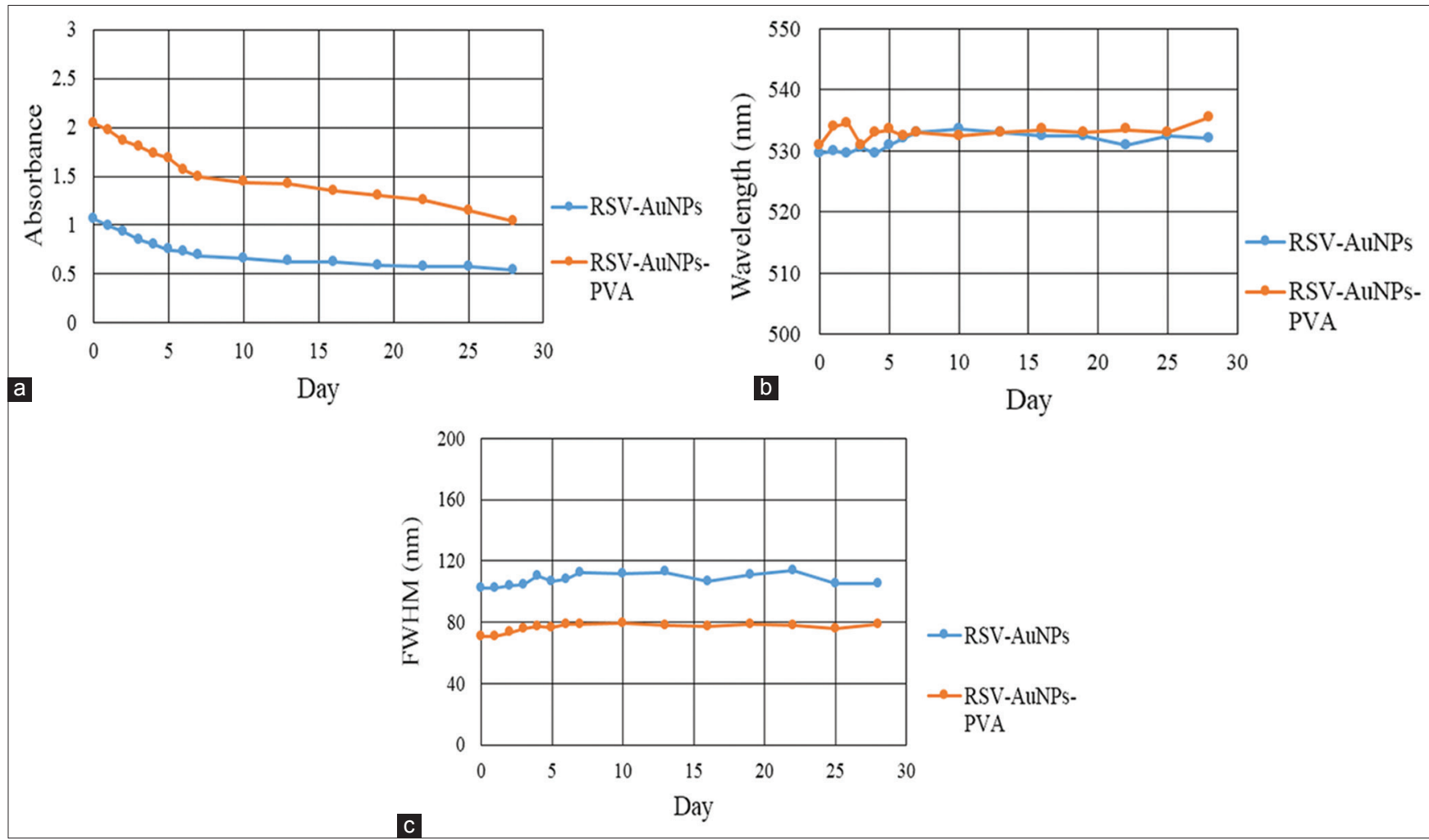

Fig. 9: (a-c) Absorbance comparison graph, maximum wavelength ( $\lambda$ max), and full width at half maximum value between resveratrol-gold nanoparticles (RSV-AuNPs) with RSV-AuNPs-polyvinyl alcohol in phosphate-buffered saline pH 7.4 


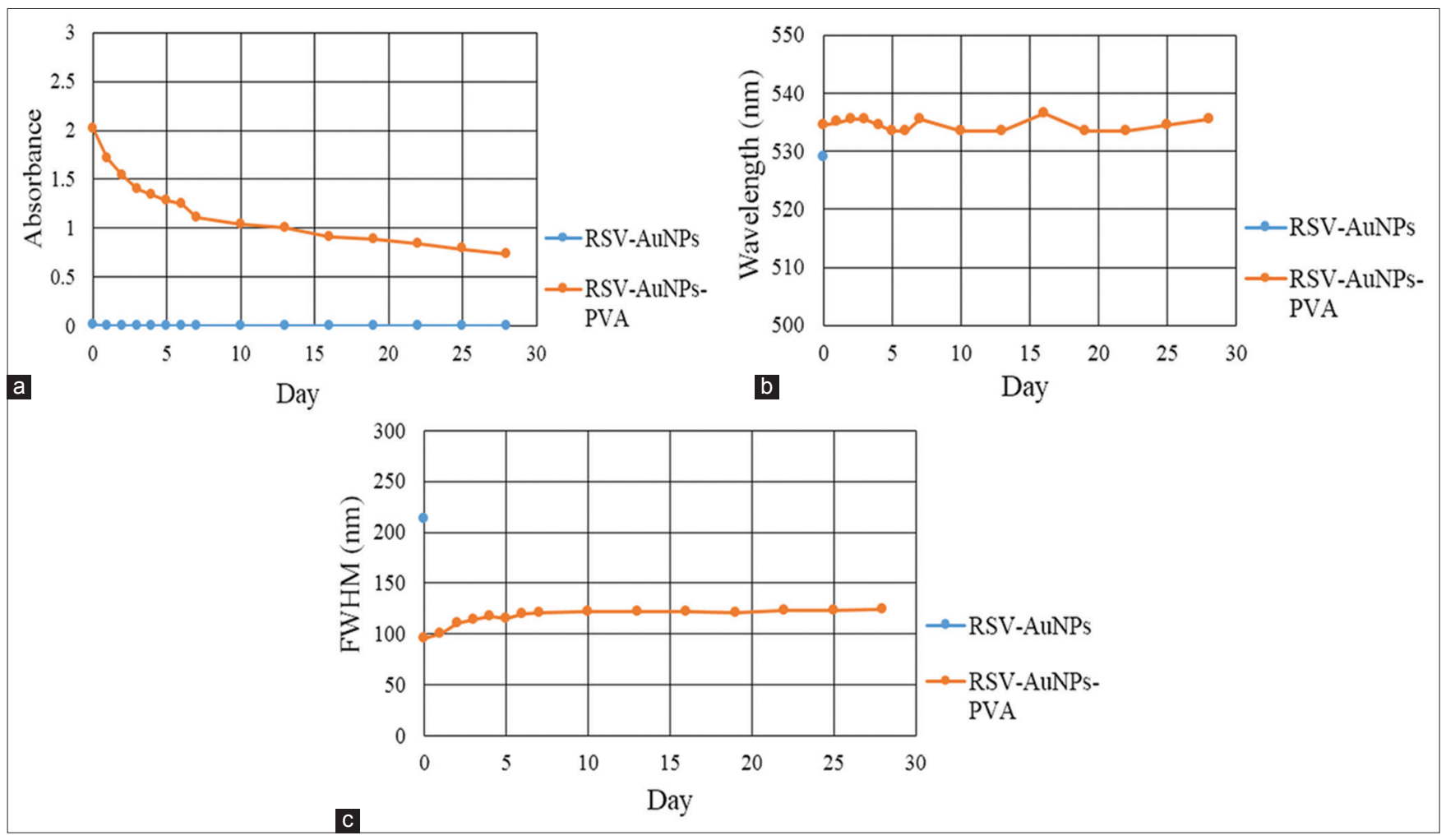

Fig. 10: (a-c) Absorbance comparison graph, maximum wavelength ( $\lambda$ max), and full width at half maximum value between resveratrolgold nanoparticles (RSV-AuNPs) with RSV-AuNPs-polyvinyl alcohol in phosphate-buffered saline pH 4
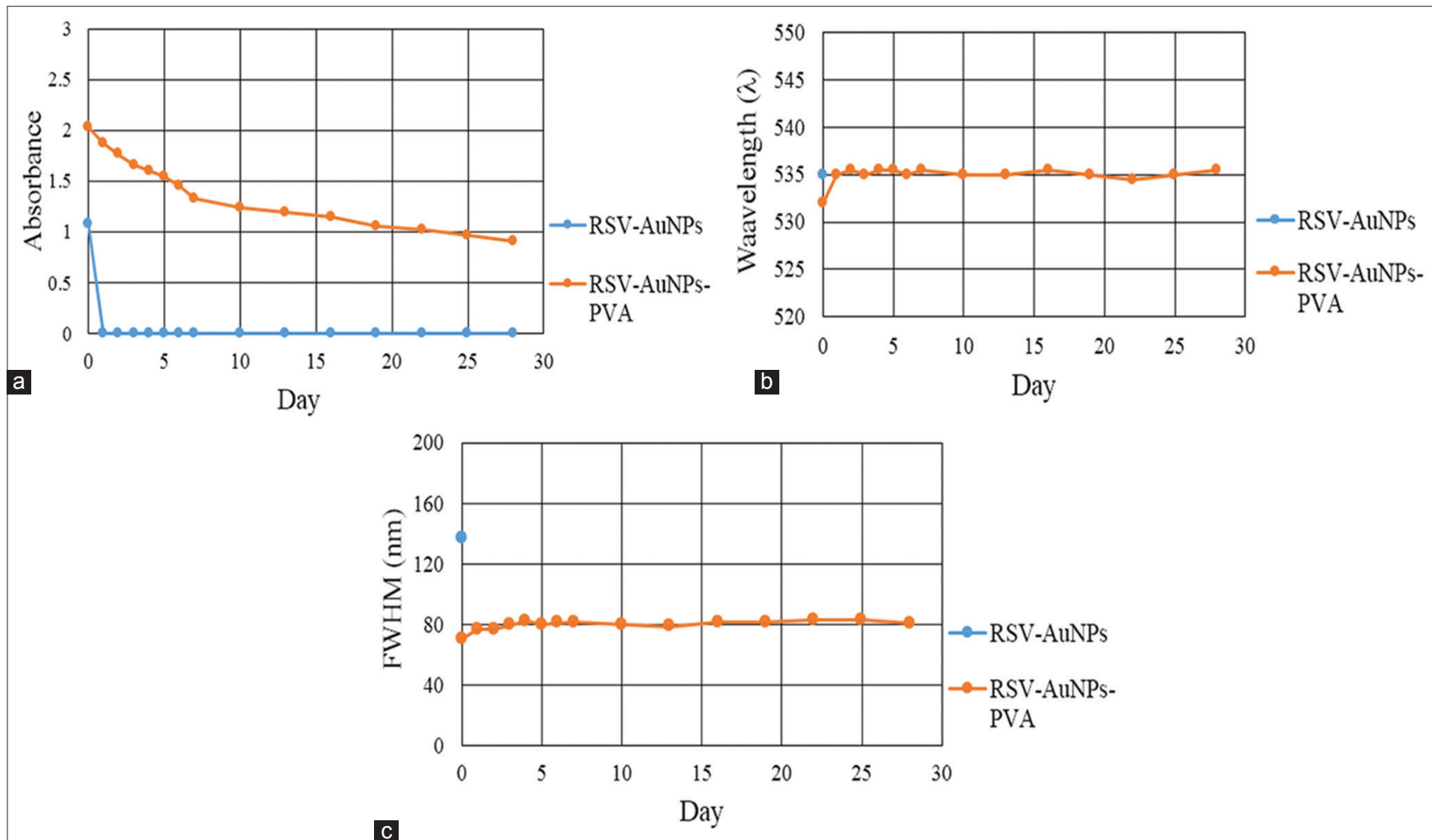

Fig. 11: (a-c) Absorbance comparison graph, maximum wavelength ( $\lambda$ max), and full width at half maximum value between resveratrolgold nanoparticles (RSV-AuNPs) with RSV-AuNPs-polyvinyl alcohol in $0.9 \% \mathrm{NaCl}$ 
The FWHM value of RSV-AuNPs was greater than RSV-AuNPs-PVA due to its widened spectrum (Fig. 10).

\section{In vitro stability analysis of RSV-AuNPs and RSV-AuNPs-PVA in PBS 0.9\% $\mathrm{NaCl}$}

The stability test of RSV-AuNPs in $0.9 \% \mathrm{NaCl}$ solution showed that RSVAuNPs were unstable in this medium (Fig. 11), demonstrated by the color change of the RSV-AuNPs solution from reddish-purple to deep purple. The next day, it turned clear with precipitates. A significant decrease in RSV-AuNPs absorbance was also observed. The maximum wavelength and FWHM value could not be calculated after day 1, because the absorption spectrum of RSV-AuNPs was flat in the region of $500-550 \mathrm{~nm}$. The RSV-AuNPs-PVA was stable in $0.9 \% \mathrm{NaCl}$ medium. Based on the spectrum, the absorbance change was relatively stable, and the wavelength shift of RSV-AuNPs-PVA was $<10 \mathrm{~nm}$. The FWHM value of RSV-AuNPs-PVA increased slightly but tended to be stable.

\section{DISCUSSION}

One of the most important aspects of the various biomedical applications of AuNPs is their stability in physiological media. Aggregation of AuNPs represents a key issue that underlies multiple deleterious effects in many therapeutic applications, including loss of efficacy, altered pharmacokinetics, and induction of unwanted immunogenicity [20]. Thus, in vitro stability tests were performed for both RSV-AuNPs and RSV-AuNPs-PVA.

Observation using UV-visible spectrophotometry can be used as a parameter of physical stability change in AuNPs because a decrease in absorbance indicates that particle size is enlarged. Furthermore, a change of $\lambda$ max $>10 \mathrm{~nm}$ during observation indicates a change in particle size of AuNPs due to aggregation of the particles [20].

FWHM is a sensitive measurement for monodispersity. Polydispersity produces wider absorbance bands and monodispersity produces narrower ones. A low FWHM value indicates monodispersity, while higher values indicate polydispersity of particles [21]. The instability of AuNPs is augmented by an increasingly widened spectrum with a high FWHM value.

In this study, in vitro stability tests of RSV-AuNPs and RSV-AuNPsPVA were performed in $2 \%$ BSA solution because albumin is the most abundant protein in human blood plasma. Stability tests using BSA are carried out to obtain an overview of the stability of a drug in human blood plasma in vitro [20].

We also conducted in vitro stability tests in a $1 \%$ cysteine solution. Cysteine was chosen because it has a sulfur atom (thiol group), otherwise known as SH. The thiol group in cysteine will bind to AuNPs with high affinity [20]. This interaction will cause aggregation of AuNPs, characterized by a change color from reddish-purple to blue [22]. The aggregation of AuNPs was also characterized by a widened spectrum [20].

At neutral $\mathrm{pH}$, the AuNPs solutions maintained their reddish-purple color, indicating no aggregation. This is because the citrate was fully deprotonated and there were negative surface charges that result in repulsion between the nearby AuNPs [23]. Thus, both RSV-AuNPs and RSV-AuNPs-PVA were stable in PBS pH 7.4.

At $\mathrm{pH}<7$, citrate is fully protonated, and thus the number of negative surface charges is greatly reduced. The decrease in negative surface charges results in aggregation and hence decreases the stability of the AuNPs [24]. In the presence of fully protonated citrate, PVA can stabilize AuNPs in pH 4 PBS. These findings indicate that RSV-AuNPs-PVA was physically stable in $\mathrm{pH} 4 \mathrm{PBS}$. Further research is needed into the impact of stabilization using PVA to the release of drugs in an AuNPs system. At $\mathrm{pH} 4$, which is the $\mathrm{pH}$ that represents cancer cells, it is expected that the drug can release quickly from the system.
$\mathrm{NaCl}$ is an electrolyte solution containing cations and anions. $\mathrm{NaCl}$ $0.9 \%$ solution can be used in biological studies and in vitro stability tests because it contains $\mathrm{Na}^{+}$and $\mathrm{Cl}^{-}$ions, which are the most abundant ions in the body's cellular system [20]. AuNPs form aggregates in the electrolyte solution, causing the particles to increase in size [20].

The results showed that RSV-AuNPs-PVA were stable in $0.9 \% \mathrm{NaCl}$ solution over the 28-day observation period, while RSV-AuNPs were only stable for $16 \mathrm{~d}$. This was presumably because PVA had formed a steric stabilization, which helped stabilize the AuNPs. However, the results of the stability tests in various media showed that RSV-AuNPsPVA were more stable in $2 \%$ BSA, PBS pH 7.4, PBS pH 4 , and $0.9 \%$ $\mathrm{NaCl}$ than RSV-AuNPs and were unstable in $1 \%$ cysteine. These results suggest that the use of PVA can improve the physical stability of RSVAuNPs conjugates.

\section{CONCLUSION}

The application of PVA to RSV-conjugated AuNPs was proficient in preventing aggregation and consequently could improve the physical stability of AuNPs. However, further optimization of other parameters, such as stirring time, temperature, and $\mathrm{pH}$, which affect the manufacture of RSV-AuNPs-PVA, is needed. Purification of the formed conjugate is also required to increase its purity and facilitate quantitative analysis.

\section{ACKNOWLEDGMENT}

We gratefully acknowledge the funding from Directorate of Research and Community Engagement Universitas Indonesia, via Hibah PITTA 2018 supporting this work.

\section{CONFLICTS OF INTEREST}

The authors declare that there are no conflicts of interest.

\section{REFERENCES}

1. Kumar CG, Poornachandra Y, Mamidyala SK. Green synthesis of bacterial gold nanoparticles conjugated to resveratrol as delivery vehicles. Colloids Surf B Biointerfaces 2014;123:311-7.

2. Kadian R. Nanoparticles: A promising drug delivery approach. Asian J Pharm Clin Res 2018;11:30-5.

3. Lavanya N, Muzib I, Jithan A, Umamahesh B. Novel nanoparticles for the oral delivery of low molecular weight heparin: In vitro and in vivo assessment. Asian J Pharm Clin Res 2017;10:254-61.

4. Kong FY, Zhang JW, Li RF, Wang ZX, Wang WJ, Wang W. Unique roles of gold nanoparticles in drug delivery, targeting and imaging applications. Molecules 2017;22:E1445.

5. Ajnai G, Chiu A, Kan T, Cheng C, Tsai T, Chang J. Trends of gold nanoparticle-based drug delivery system in cancer therapy. J Exp Clin Med 2014;6:172-8.

6. Sharma N, Bhatt G, Kothiyal P. Gold nanoparticles synthesis, properties, and forthcoming applications-a review. Indian J Pharm Biol Res 2015;3:13-27.

7. Kumar A, Mansour H, Friedman A, Blough E. Nanomedicine in Drug Delivery. Boca Raton: CRC Press; 2017.

8. Rahme K, Chen L, Hobbs RG, Morris MA, O’Driscoll C, Holmes JD. PEGylated gold nanoparticles: Polymer quantification as a function of PEG lengths and nanoparticle dimensions. RSC Adv 2013;3:6085-94.

9. Pacławski K, Streszewski B, Jaworski W, Luty-Błocho M, Fitzner K. Gold nanoparticles formation via gold (III) chloride complex ions reduction with glucose in the batch and in the flow microreactor systems. Colloids Surf Physicochem Eng Asp 2012;413:208-15.

10. Gaikwad P, Sharma S, Sudarshan K, Kumar V, Kshirsagar A, Pujari P. Molecular packing of polyvinyl alcohol in PVA-gold nanoparticles composites and its role on thermo-mechanical properties. Polym Compos 2016;39:1-6.

11. Park SY, Chae SY, Park JO, Lee KJ, Park G. Gold-conjugated resveratrol nanoparticles attenuate the invasion and MMP-9 and COX2 expression in breast cancer cells. Oncol Rep 2016;35:3248-56.

12. Souto AA, Carneiro MC, Seferin M, Senna MJ, Conz A, Gobbi K. Determination of trans-resveratrol concentrations in Brazilian red wines by HPLC. J Food Compost Anal 2001;14:441-5.

13. Djajadisastra J, Sutriyo S, Purnamasari P, Pujiyanto A. Antioxidant activity of gold nanoparticles using gum Arabic as a stabilizing agent. 
Int J Pharm Pharm Sci 2014;6:462-5.

14. Zhang Y, Chu W, Foroushani AD, Wang H, Li D, Liu J, et al. New gold nanostructures for sensor applications: A review. Materials (Basel) 2014; 7:5169-201.

15. Esumi K, Suzuki A, Yamahira A, Torigoe K. Role of poly (amidoamine) dendrimers for preparing nanoparticles of gold, platinum, and silver. Langmuir 2000;16:2604-8.

16. Pertiwi RD, Djajadisastra J, Mutalib A, Pujiyanto A. Preparation of gold nanoparticles with based on conjugated gum Arabic vincristine and evaluation of their in vitro characteristics. J Ilmu Kefarmasian Indones 2018;16:6-11.

17. Avadi MR, Sadeghi AM, Mohammadpour N, Abedin S, Atyabi F, Dinarvand $\mathrm{R}$, et al. Preparation and characterization of insulin nanoparticles using chitosan and Arabic gum with ionic gelation method. Nanomedicine 2010;6:58-63.

18. Mohanraj VJ, Chen Y. Nanoparticles a review. Trop J Pharm Res 2006;5:561-73.
19. Chen W. What is Zeta Potential? San Diego: The American Filtration and Separations Society; 2016.

20. Kattumuri V. Gold nanoparticles for biomedical applications: Synthesis, characterization, in vitro and in vivo studies. USA: Dissertation the Faculty of Graduate School, University of Missouri, Columbia; 2006.

21. Oliveira J, Prado A, Keijok W, Ribeiro M, Pontes M, Nogueira B, et al. A helpful method for controlled synthesis of monodisperse gold nanoparticles through response surface modeling. Arab J Chem 2017;1-11

22. Vaseghi A, Safaie N, Bakhshinejad B, Mohsenifar A, Sadeghizadeh M. Detection of Pseudomonas syringae pathovars by thiol-linked DNAgold nanoparticle probes. Sens Actuators B Chem 2013;181:644-51

23. Wagers K, Chui T, Adem S. Effect of $\mathrm{pH}$ on the stability of gold nanoparticles and their application for melamine detection in infant formula. IOSR J Appl Chem 2014;7:15-20.

24. Butt H, Graf K, Kappl M. Physics and Chemistry of Interfaces. Weinheim: Wiley-VCH; 2006. 